\title{
Kyphosis Progression after Balloon Kyphoplasty Compared with Conservative Treatment
}

\author{
Keiichiro Iida ${ }^{1}$, Katsumi Harimaya ${ }^{1}$, Kiyoshi Tarukado ${ }^{1}$, \\ Osamu Tono ${ }^{1}$, Yoshihiro Matsumoto ${ }^{2}$, Yasuharu Nakashima ${ }^{2}$ \\ ${ }^{1}$ Department of Orthopaedic Surgery, Kyushu University Beppu Hospital, Beppu, Japan \\ ${ }^{2}$ Department of Orthopaedic Surgery, Faculty of Medical Sciences, Kyushu University, Fukuoka, Japan
}

Study Design: Retrospective cohort study (level of evidence: 4).

Purpose: To demonstrate the effects of balloon kyphoplasty (BKP) on prevention of kyphosis progression.

Overview of Literature: Kyphoplasty can correct local kyphosis (fractured vertebra), but its efficacy is attenuated by adjacent fracture and/or disc height reduction with endplate damage. With these attenuating factors incorporated, a comparison between BKP and conservative treatment is necessary to verify the effect of BKP on kyphosis correction.

Methods: This study retrospectively analyzed 129 patients treated conservatively and 95 treated with BKP in our institution. The rate of new adjacent fracture occurrence was determined using Kaplan-Meier analysis. We examined the progression of local kyphosis in patients who underwent lateral X-ray after 1 year. Local kyphosis was measured as an angle between higher end plate of the vertebra above and lower end plate of the vertebra below the fractured vertebra. The comparison included 45 patients treated conservatively and 58 treated with BKP.

Results: The incidence of new adjacent fracture at 1 year was $7.3 \%$ in the conservative treatment group and $23.2 \%$ in the BKP group $(p<0.001)$, while the progression of local kyphosis at 1 year was $5.7^{\circ} \pm 4.7^{\circ}$ and $3.2^{\circ} \pm 4.6^{\circ}$, respectively $(p=0.01)$. Relative to conservative treatment, local kyphosis did not progress after BKP, despite the higher incidence of adjacent fracture. Local kyphosis progressed in cases with adjacent fracture compared with those without adjacent fracture $\left(6.0^{\circ} \pm 4.3^{\circ} \mathrm{vs} .2 .1^{\circ} \pm 4.3^{\circ}, p=0.003\right)$, and fractured vertebral instability was found to be a risk factor for adjacent fracture in BKP.

Conclusions: Compared with the conservative treatment, BKP suppressed the progression of local kyphosis. However, an adjacent fracture attenuated the BKP correction and was more frequently seen in patients with unstable vertebral fractures.

Keywords: Vertebral fracture; Adjacent fracture; Local kyphosis; Balloon kyphoplasty

\section{Introduction}

Kyphoplasty can offer faster pain relief and improved functional outcomes compared with conservative treat- ment [1-4]. Reports have shown that the difference in the clinical outcome between kyphoplasty and conservative treatment decreases over time, and that most patients with vertebral fracture are cured with bone fusion and achieve

Received Dec 13, 2018; Revised Feb 20, 2019; Accepted Feb 23, 2019

Corresponding author: Keiichiro Iida

Department of Orthopaedic Surgery, Kyushu University Beppu Hospital, 4546 Tsurumibaru, Beppu, Oita, 874-0838, Japan

Tel: +81-977-27-1600, Fax:+81-977-27-1605, E-mail: iida-k16@ortho.med.kyushu-u.ac.jp 
pain relief without surgical intervention [1-4]. However, we often observe a development of persistent kyphosis after vertebral fracture, even in patients without back pain. This persistent local kyphosis can cause postural abnormalities and future back pain because of back muscle fatigue.

The effects of kyphoplasty on kyphosis correction have been widely reported, but a comparison between balloon kyphoplasty (BKP) and conservative treatment has not been made. Kyphoplasty can correct kyphosis due to a fractured vertebra, but the correction can be attenuated by an adjacent vertebral fracture and/or decreased disc height due to endplate damage. Therefore, a comparison between $\mathrm{BKP}$ and conservative treatment, including these attenuating factors, is necessary to verify the effect of BKP in correcting kyphosis.

We investigated the rate of adjacent vertebral fracture and compared the progression of local kyphosis in BKP and conservative treatment groups to demonstrate the ability of kyphoplasty to suppress kyphosis.

\section{Materials and Methods}

\section{Patients}

This study was approved by the Kyushu University institutional review board (IRB approval no., 28-324). It was determined that written informed consent wouldn't be needed due to the retrospective nature of the study. Patients with vertebral fractures who underwent conservative treatment or BKP between 2013 and 2017 were retrospectively analyzed. Patients with pathological fractures or fractures with neurological symptoms were excluded. The fracture level was T5 to L5, and all fractures were confirmed by instability between sitting and supine positions and bone signal intensity changes on magnetic resonance imaging (MRI). To determine the incidence of new adjacent fractures, we excluded the patients with collapsed vertebrae on both sides of the fractured vertebra even if these had already healed, because new fractures in collapsed vertebrae are difficult to detect.

We investigated the rate of new adjacent fracture using Kaplan-Meier analysis in 129 patients treated conservatively and 95 patients treated with BKP. Age, sex, and fracture site of the patients were recorded. The duration of corset use and bed-rest time were dependent on patient's condition. BKP was performed under general anesthesia, and patients treated with BKP tended to get out of bed the next day with a soft corset. New adjacent fracture was confirmed by instability between sitting and supine position and/or a 15\% loss of height and/or bone signal intensity change on MRI.

We compared the progression of local kyphosis in patients who underwent lateral X-ray in supine position 1 year after the treatment. This comparison included 45 patients treated conservatively (eight with adjacent fractures) and 58 patients treated with BKP (16 with adjacent fractures). The progression of local kyphosis between patients with and without adjacent fracture was also measured. Age, sex, fracture site, and instability of the fracture determining the condition of the fractured vertebra before treatment were investigated. Fracture instability was calculated by the change in angle of the fractured vertebral endplate between sitting and supine positions (Fig. 1). Local kyphosis was measured as the angle between the higher endplate of the upper vertebral body and the lower endplate of the lower vertebral body of the fractured vertebra in supine position, including kyphosis of the adjacent fracture (Fig. 1).

Age, sex, fracture site, fracture instability, cement amount, and presence of cement leak were investigated in 71 patients who were followed up for more than 1 year to identify the risk factors for adjacent vertebral fracture after BKP.

\section{Statistical analyses}

The chi-square test and Wilcoxon's signed-rank test were used for group comparisons. The rates without adjacent fracture were calculated with the Kaplan-Meier method and compared using Wilcoxon's test. To clarify the prognostic risk factors for adjacent fracture, multivariate statistical analysis was performed. Factors included in the multivariate model were age, sex, fracture site, fracture instability, cement amount, and presence of cement leak. A $p<0.05$ was considered significant for all statistical tests.

\section{Results}

Adjacent vertebral fractures occurred more often in the BKP group than in the conservative treatment group $(p<0.001)$ (Fig. 2). Patient details are shown in Table 1. Patients treated with BKP were significantly older $(77.3 \pm 8.1$ years versus $80.1 \pm 6.7$ years, $p=0.01$ ) than those in the 

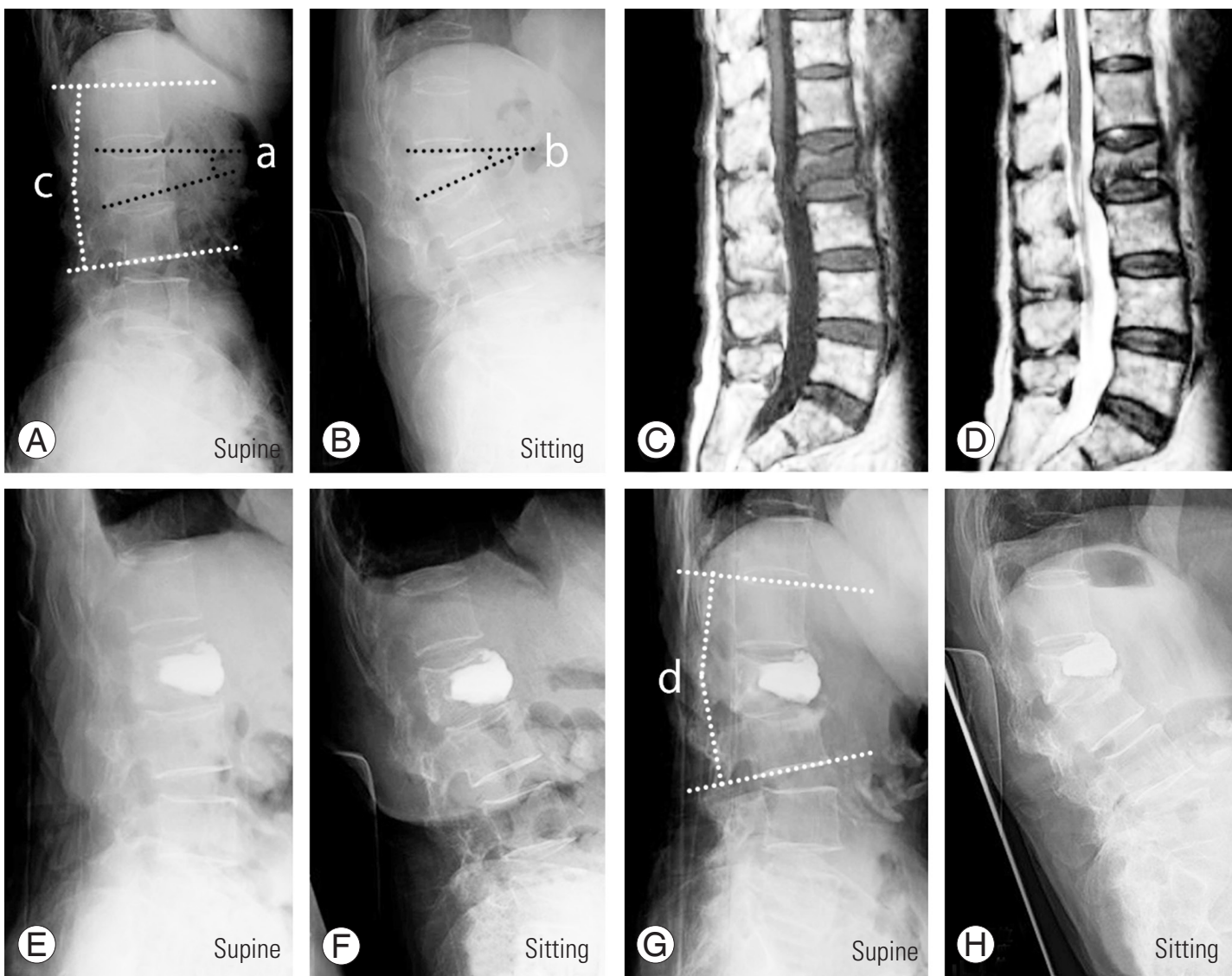

Instability of factured vertebra=(b-a) degree

Progression of kyphosis=(d-c) degree

Fig. 1. BKP-treated patient. Lateral $X$-ray in supine (A) and sitting positions (B), and T1 (C) and T2 (D) magnetic resonance imaging when the patient visited our institution. Lateral X-ray in supine (E) and sitting positions (F) 1 week after BKP. Lateral $X$-ray in supine (G) and sitting positions (H) 1 year after the treatment. Kyphosis measured $16^{\circ}$ in supine position and $25^{\circ}$ in sitting position when the patient visited our institution. Instability of the fractured vertebra was $9^{\circ}$. Local kyphosis measured $11^{\circ}$ before treatment and $20^{\circ} 1$ year after BKP. The progression of local kyphosis was $9^{\circ}$. BKP, balloon kyphoplasty.

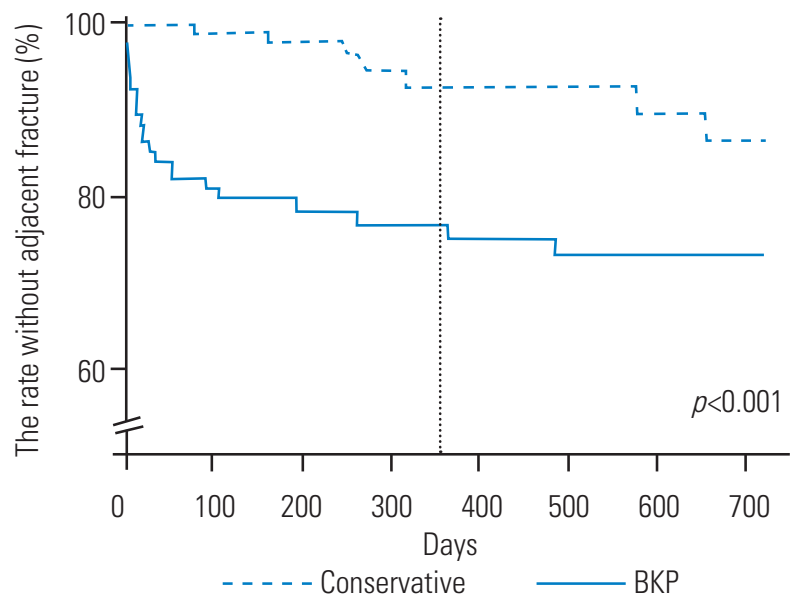

Fig. 2. Kaplan-Meier analysis of adjacent fractures among patients treated conservatively or with BKP. BKP, balloon kyphoplasty.
Table 1. Details of BKP- and conservatively-treated patients according to the presence of adjacent fracture

\begin{tabular}{lccc} 
Variable & $\begin{array}{c}\text { Conservative } \\
\text { group }(\mathrm{n}=129)\end{array}$ & $\begin{array}{c}\text { BKP group } \\
(\mathrm{n}=95)\end{array}$ & $p$-value \\
\hline Age (yr) & $77.3 \pm 8.1$ & $80.1 \pm 6.7$ & 0.01 \\
\hline Sex & & & 0.54 \\
\hline Male & 34 & 28 & \\
\hline Female & 95 & 67 & \\
Site of fracture (\%) & & & \\
\hline T5-10 & 8 & 8 & \\
\hline T11-L1 & 58 & 54 & \\
\hline L2-5 & 34 & 38 \\
\hline
\end{tabular}

Values are presented as mean \pm standard deviation or number. BKP, balloon kyphoplasty. 
Table 2. Progression of kyphosis in BKP- and conservatively-treated patients

\begin{tabular}{lccc} 
Variable & Conservative group $(n=45 ; 8$ adjacent fracture $)$ & BKP group $(n=58 ; 16$ adjacent fracture $)$ & $p$-value \\
Progression of kyphosis & & & \\
Adjacent fracture - & $5.1 \pm 4.7$ & $6.1 \pm 4.3$ & 0.0044 .3 \\
\hline Adjacent fracture + & $8.3 \pm 4.5$ & $3.2 \pm 4.6$ & 0.27 \\
\hline Total & $5.7 \pm 4.7$ & 0.01 \\
\hline
\end{tabular}

Values are presented as mean \pm standard deviation. BKP, balloon kyphoplasty.

Table 3. Details of BKP- and conservatively-treated patients according to progression of local kyphosis

\begin{tabular}{|c|c|c|c|}
\hline Variable & Conservative group ( $n=45 ; 8$ adjacent fracture) & BKP group ( $n=58 ; 16$ adjacent fracture) & $p$-value \\
\hline Age (yr) & $77.8 \pm 12.6$ & $80.6 \pm 6.8$ & 0.04 \\
\hline Sex & & & 0.39 \\
\hline Male & 16 & 16 & \\
\hline Female & 29 & 42 & \\
\hline \multicolumn{4}{|l|}{ Site of fracture (\%) } \\
\hline $\mathrm{T} 11-\mathrm{L} 1$ & 60 & 62 & \\
\hline L2-5 & 40 & 38 & \\
\hline Instability of vertebra & $5.5 \pm 4.2$ & $8.1 \pm 5.8$ & 0.03 \\
\hline
\end{tabular}

Values are presented as mean \pm standard deviation or number. BKP, balloon kyphoplasty.

conservative treatment group. The incidence of adjacent fracture after 1 year was $7.3 \%$ in the conservative treatment group and $23.2 \%$ in the BKP group (Fig. 2).

The progression of local kyphosis was $5.7^{\circ} \pm 4.7^{\circ}$ in the conservative treatment group and $3.2^{\circ} \pm 4.6^{\circ}$ in the BKP group at 1 -year follow-up $(p=0.01)$. Local kyphosis in the patients without adjacent fracture progressed less significantly in the BKP group $\left(5.1^{\circ} \pm 4.7^{\circ}\right.$ versus $2.1^{\circ} \pm 4.3^{\circ}$, $p=0.004$ ) (Table 2). The calculation methods are shown in Fig. 1. Patient details comparing the degrees of local kyphosis are shown in Table 3 . The number of patients decreased compared to those shown in Table 1 because assessment was performed at fixed points in time, as kyphosis progressed with time and patients lacking lateral X-ray findings at 1 year after treatment could not be included. Among 95 patients treated with BKP, three patients underwent additional BKP for an adjacent fracture, while other three patients underwent reoperation for instability or infection of the operated vertebral fracture. These six patients were not included in the comparison of local kyphosis. Patients treated with BKP were significantly older $(77.8 \pm 12.6$ years versus $80.6 \pm 6.8$ years, $p=0.04)$ than those in the conservative treatment group. Instability of the fractured vertebra was significantly greater in patients treated with BKP than in those treated with conservative treatment $\left(5.5^{\circ} \pm 4.2^{\circ}\right.$ versus $\left.8.1^{\circ} \pm 5.8^{\circ}, p=0.03\right)$.

In a comparison between patients treated with BKP, local kyphosis progressed in those with adjacent fracture compared with those without adjacent fracture $\left(6.0^{\circ} \pm 4.3^{\circ}\right.$ versus $2.1^{\circ} \pm 4.3^{\circ}, p=0.003$ ) (Table 2 ), and instability of the fractured vertebra was found to be a risk factor in those with adjacent fracture compared to those without adjacent fracture $(p=0.046)$. There was no significant difference in the cement amount or leakage between the 2 groups (Table 4).

\section{Case 1: balloon kyphoplasty-treated patient}

An 87-year-old woman with persistent back pain was referred to our institution. She experienced back pain while exercising and the pain had persisted. She had a $9^{\circ}$ of spinal instability between sitting and supine positions, and vertebral fracture was detected on MRI at the second lumbar vertebra. She underwent BKP 40 days after the injury. The cement amount was $9 \mathrm{~mL}$, and there was no leak. At the 1-year follow-up, the treated vertebra was compressed 
Table 4. Details of balloon kyphoplasty- and conservatively-treated patients

\begin{tabular}{|c|c|c|c|c|}
\hline \multirow{2}{*}{ Variable } & \multirow{2}{*}{ Adjacent fracture - $(n=48)$} & \multirow{2}{*}{ Adjacent fracture $+(n=23)$} & \multicolumn{2}{|c|}{$p$-value } \\
\hline & & & Univariate & Multivariate \\
\hline Age (yr) & $80.5 \pm 6.7$ & $81.2 \pm 6.8$ & 0.62 & 0.41 \\
\hline Sex & & & 0.78 & 0.17 \\
\hline Male & 15 & 6 & & \\
\hline Female & 33 & 17 & & \\
\hline Amount of cement & $8.0 \pm 5.5$ & $9.4 \pm 3.8$ & 0.12 & 0.11 \\
\hline Cement leak & $(+) 21,(-) 27$ & $(+) 13,(-) 10$ & 0.45 & 0.21 \\
\hline Site of fracture $(\%)$ & & & & 0.55 \\
\hline T5-10 & 4 & 8 & & \\
\hline $\mathrm{T} 11-\mathrm{L} 1$ & 61 & 52 & & \\
\hline L2-5 & 35 & 40 & & \\
\hline Instability of vertebra & $7.2 \pm 4.6$ & $10.3 \pm 5.5$ & 0.049 & 0.046 \\
\hline
\end{tabular}

Values are presented as mean \pm standard deviation or number.

and the adjacent disc height had decreased. The progression of kyphosis was $9^{\circ}$. Local kyphosis had progressed in spite of restoration of fractured vertebral height by BKP (Fig. 1).

\section{Case 2: balloon kyphoplasty-treated patient with ad- jacent fracture}

A 78-year-old woman with idiopathic back pain visited our institution. She had a history of rheumatoid arthritis and had been treated with tocilizumab and prednisolone. A second lumbar vertebral fracture was detected and treated conservatively with hospitalization (Fig. 3A-C). She underwent pacemaker replacement for sick sinus syndrome during her stay at the hospital. She also underwent BKP due to persistent back pain 73 days after admission and reported immediate pain relief (Fig. 3D). She complained of idiopathic back pain and was found to have a first lumbar vertebral fracture 8 days after BKP (Fig. 3E). The collapse progressed, and another BKP was performed 14 days after the first BKP. She was discharged from our institution without back pain 1 month after the second BKP treatment. Two days after discharge, she returned to our hospital with back pain. A 12th thoracic vertebral fracture was detected and treated conservatively with admission (Fig. 3F). The collapse progressed, and BKP was performed 2 months after the injury. Ten days after the third BKP treatment, an 11th thoracic vertebral fracture was detected (Fig. 3G). She was treated conservatively and discharged
1 month after the third BKP treatment. No vertebral fractures had occurred after 2 years of follow-up (Fig. 3H).

\section{Discussion}

We found that local kyphosis did not progress in patients treated with BKP compared with those treated conservatively at 1-year follow-up. Many reports have described the effect of kyphosis correction, but none have compared the effects of BKP with those of conservative treatment. BKP can correct kyphosis caused by a fractured vertebra, but the correction can be attenuated by adjacent vertebral fractures and/or decreases in disc height due to endplate damage. Pradhan et al. [5] reported that angular reduction produced by kyphoplasty at the level of the fracture does not translate to similar corrections in the spinal segments spanned by adjacent vertebrae. The intervertebral disc absorbs a significant amount of the correction achieved by kyphoplasty. Since the degree of reduction in adjacent segmental alignment depends on both vertebral fracture and disc condition, the effect of correcting local kyphosis was measured by the endplates across the intervertebral disc.

Adjacent fracture is another problem associated with kyphosis correction. Adjacent fracture occurs more frequently in BKP, typically within 2 months of the procedure. Adjacent fracture within a few months is known to be a major complication [6,7], but a systematic review concluded that the rate of adjacent fracture was not mark- 

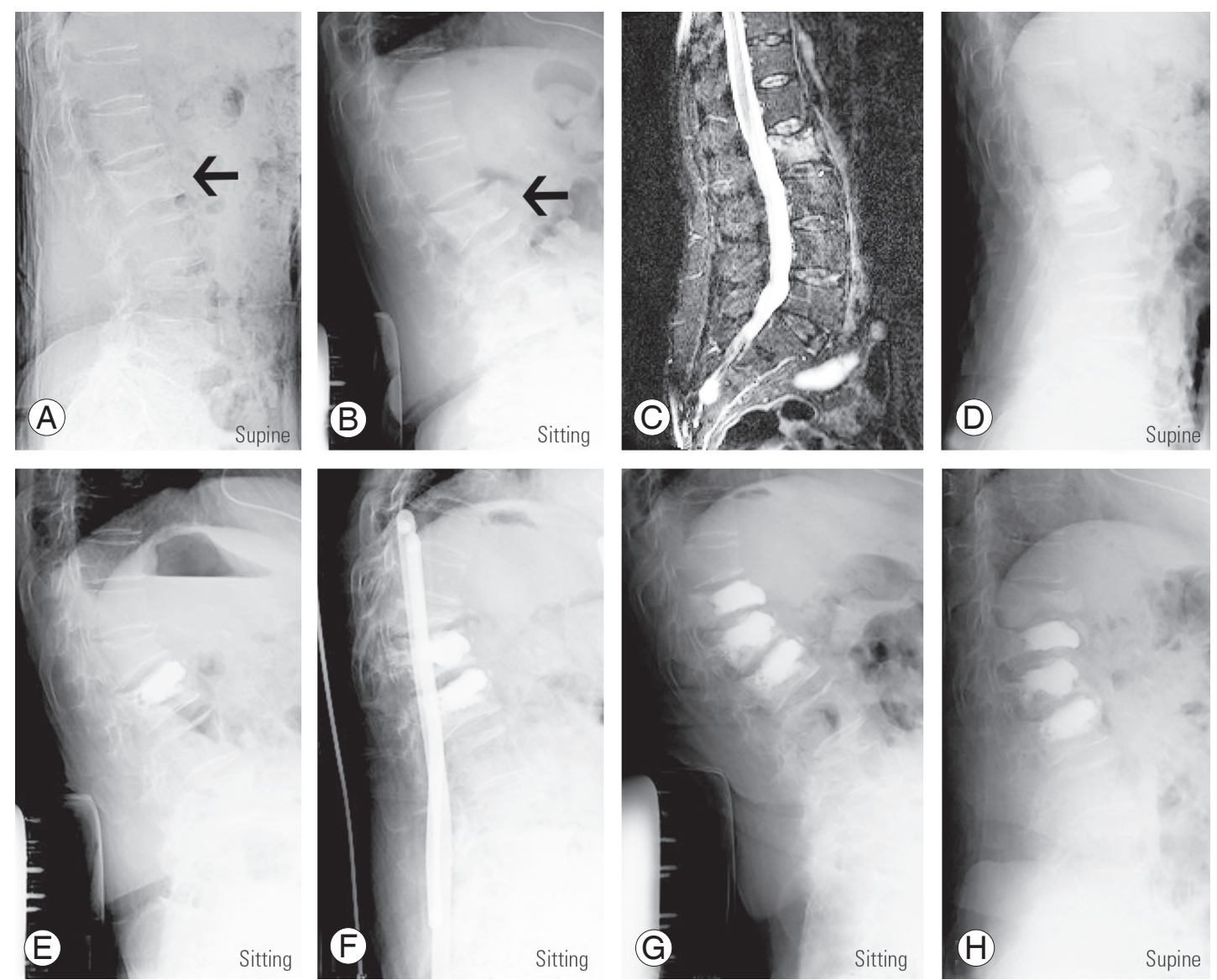

Fig. 3. BKP-treated patient who developed an adjacent fracture. Lateral X-ray in supine (A) and sitting positions (B) and bone edema (C) on T2 fat-suppression magnetic resonance imaging when the patient visited our institution. The black arrow indicates a first lumbar vertebral fracture. (D) Lateral X-ray in supine position when she underwent BKP. Lateral X-ray in sitting position when she developed first (E), second (F), and third (G) adjacent vertebral fractures. $(\mathbf{H})$ Lateral X-ray in supine position 1 year after the third adjacent vertebral fracture. BKP, balloon kyphoplasty.

edly different between BKP and conservative treatment $[8,9]$. We believe that this discrepancy in fracture rate may be partly due to the method used for fracture detection. We performed MRI in all patients before treatment and restricted the selection of patients to those with adjacent vertebra on at least one side having no history of fracture. In addition, there were new adjacent fractures that could not be detected by simple X-rays. Such fractures could only be detected by comparing the X-ray findings in sitting and supine positions or by MRI. Regarding the rate of new vertebral fracture, our study concluded that adjacent fracture was indeed a complication associated with BKP. For these reasons, we assessed the progression of local kyphosis using the angle between the higher endplate of the upper vertebral body and the lower endplate of the lower vertebral body of the fractured vertebra in supine position and compared the findings between BKP and conserva- tive treatment groups. We selected supine rather than standing position to compare kyphosis angle because we had to exclude the effect of back pain on posture. The kyphosis angle was influenced by back pain when measured in standing position, especially in early vertebral fracture cases. Therefore, we expected this result to be somewhat different in standing position, as the adjacent disc height reduction might be clearer with loading.

Surgical intervention for the fractured vertebra using BKP suppressed the progression of kyphosis, but the correction was attenuated by the presence of an adjacent fracture. We identified fractured vertebral instability as a risk factor, but the degree of restoration of fractured vertebral height and cement leakage have been reported as risk factors for adjacent fracture [10-12]. We found no significant difference in the cement amount or leakage between patients with and without adjacent fractures, as these 
factors were dependent on the surgeon's plan and skill. However, both the degree of restoration of fractured vertebral height or cement leakage and the instability of the fractured vertebra indicated that more the severity of vertebral damage, greater the risk of adjacent fracture when BKP was performed. In the meta-analysis of prospective trials, the insignificant difference in the rate of adjacent fracture may due to the condition of the recently injured patients, wherein the fractured vertebra was relatively well preserved. Early intervention may reduce the risk of adjacent fracture and provide a good clinical outcome, but it can also include patients who were considered suitable candidates for conservative treatment.

We were unable to determine the clinical significance of the suppression of progression of local kyphosis. Local kyphosis itself can lead to postural abnormalities and an increased risk of falling [13,14]. Global balance was reported to be theoretically related to back pain due to muscle fatigue [15], so whether suppressing local kyphosis due to vertebral fracture influences global balance is a matter of concern. Local kyphosis of the fractured vertebra is compensated for by hyperextension or hypolordosis of another spinal segment but can cause global imbalance if insufficient [16]. Suppressing local kyphosis using BKP may reduce these compensatory changes in another spinal segment and future back pain induced by global imbalance. Some reports found that correcting fractured vertebrae using kyphoplasty did not influence the global balance [17], but arrived at this conclusion without comparing BKP to conservative treatment. Further studies are needed to clarify the influence of correcting local kyphosis using BKP on global balance.

Our study showed selection bias. We identified patients for BKP treatment among those with persistent back pain or spinal instability who were receiving conservative treatment. Therefore, the instability of the fractured vertebra before treatment was investigated to determine its condition, as this was a major requirement for comparing the outcomes of the two treatment approaches. Although the condition of the fractured vertebra was worse in patients treated with BKP than in those treated conservatively, local kyphosis was not as advanced in the BKP patients. Prospective studies under equal conditions are necessary to ensure an accurate comparison of the outcomes; however, we believe that this study demonstrated the efficacy of BKP in kyphosis suppression. The lack of information on bone mineral density (BMD) and anti-osteoporosis treatments was another problem affecting the accurate comparison of the outcomes. We performed BKP for patients with persistent back pain or spinal instability, and the BMD values did not influence the treatment decisions. The use of bisphosphonates after injuries reportedly do not affect patient outcomes and vertebral collapse [18]. However, we must consider the possibility that restricting external fixation and using strong medication for osteoporosis was performed in the patients treated with BKP to prevent adjacent fracture.

In summary, local kyphosis did not progress after BKP compared with that of conservative treatment despite the higher incidence of adjacent fracture. For further improvements in outcomes, a decrease in the incidence rate of adjacent fractures is required. Performing BKP for severely unstable vertebrae may results in worse outcomes.

\section{Conclusions}

In this retrospective series, BKP suppressed the progression of local kyphosis relative to conservative treatment. However, the presence of an adjacent fracture attenuated the correction provided by BKP, and adjacent fractures occurred more frequently in patients with unstable vertebral fractures.

\section{Conflict of Interest}

No potential conflict of interest relevant to this article was reported.

\section{Acknowledgments}

We would like to thank Editage (www.editage.jp) for English language editing.

\section{References}

1. Wardlaw D, Cummings SR, Van Meirhaeghe J, et al. Efficacy and safety of balloon kyphoplasty compared with non-surgical care for vertebral compression fracture (FREE): a randomised controlled trial. Lancet 2009;373:1016-24.

2. Klazen CA, Lohle PN, de Vries J, et al. Vertebroplasty versus conservative treatment in acute osteoporotic vertebral compression fractures (Vertos II): an openlabel randomised trial. Lancet 2010;376:1085-92. 
3. Yang EZ, Xu JG, Huang GZ, et al. Percutaneous vertebroplasty versus conservative treatment in aged patients with acute osteoporotic vertebral compression fractures: a prospective randomized controlled clinical study. Spine (Phila Pa 1976) 2016;41:653-60.

4. Alvarez L, Alcaraz M, Perez-Higueras A, et al. Percutaneous vertebroplasty: functional improvement in patients with osteoporotic compression fractures. Spine (Phila Pa 1976) 2006;31:1113-8.

5. Pradhan BB, Bae HW, Kropf MA, Patel VV, Delamarter RB. Kyphoplasty reduction of osteoporotic vertebral compression fractures: correction of local kyphosis versus overall sagittal alignment. Spine (Phila Pa 1976) 2006;31:435-41.

6. Uppin AA, Hirsch JA, Centenera LV, Pfiefer BA, Pazianos AG, Choi IS. Occurrence of new vertebral body fracture after percutaneous vertebroplasty in patients with osteoporosis. Radiology 2003;226:11924.

7. Fribourg D, Tang C, Sra P, Delamarter R, Bae H. Incidence of subsequent vertebral fracture after kyphoplasty. Spine (Phila Pa 1976) 2004;29:2270-6.

8. Papanastassiou ID, Phillips FM, Van Meirhaeghe J, et al. Comparing effects of kyphoplasty, vertebroplasty, and non-surgical management in a systematic review of randomized and non-randomized controlled studies. Eur Spine J 2012;21:1826-43.

9. Zhang H, Xu C, Zhang T, Gao Z, Zhang T. Does percutaneous vertebroplasty or balloon kyphoplasty for osteoporotic vertebral compression fractures increase the incidence of new vertebral fractures?: a metaanalysis. Pain Physician 2017;20:E13-28.

10. Kim MH, Lee AS, Min SH, Yoon SH. Risk factors of new compression fractures in adjacent vertebrae after percutaneous vertebroplasty. Asian Spine J 2011;5:180-7.
11. Komemushi A, Tanigawa N, Kariya S, et al. Percutaneous vertebroplasty for osteoporotic compression fracture: multivariate study of predictors of new vertebral body fracture. Cardiovasc Intervent Radiol 2006;29:580-5.

12. Kim SH, Kang HS, Choi JA, Ahn JM. Risk factors of new compression fractures in adjacent vertebrae after percutaneous vertebroplasty. Acta Radiol 2004;45:440-5.

13. Kim DH, Choi DH, Park JH, Lee JH, Choi YS. What is the effect of spino-pelvic sagittal parameters and back muscles on osteoporotic vertebral fracture? Asian Spine J 2015;9:162-9.

14. Van der Jagt-Willems HC, de Groot MH, van Campen JP, Lamoth CJ, Lems WF. Associations between vertebral fractures, increased thoracic kyphosis, a flexed posture and falls in older adults: a prospective cohort study. BMC Geriatr 2015;15:34.

15. Glassman SD, Bridwell K, Dimar JR, Horton W, Berven S, Schwab F. The impact of positive sagittal balance in adult spinal deformity. Spine (Phila Pa 1976) 2005;30:2024-9.

16. Booth KC, Bridwell KH, Lenke LG, Baldus CR, Blanke KM. Complications and predictive factors for the successful treatment of flatback deformity (fixed sagittal imbalance). Spine (Phila Pa 1976) 1999;24:171220.

17. Kanayama M, Oha F, Iwata A, Hashimoto T. Does balloon kyphoplasty improve the global spinal alignment in osteoporotic vertebral fracture? Int Orthop 2015;39:1137-43.

18. Hoshino M, Tsujio T, Terai $\mathrm{H}$, et al. Impact of initial conservative treatment interventions on the outcomes of patients with osteoporotic vertebral fractures. Spine (Phila Pa 1976) 2013;38:E641-8. 